\title{
Double, triple and quadruple cannulation for veno-arterial extracorporeal membrane oxygenation support: is there a limit?
}

\author{
Daniele Camboni ${ }^{1}$, Alois Philip ${ }^{1}$, Christof Schmid ${ }^{1}$, Antonio Loforte ${ }^{2}$ \\ ${ }^{1}$ Department of Cardiothoracic Surgery, University Medical Center Regensburg, Regensburg, Germany; ${ }^{2}$ Department of Cardiovascular Surgery and \\ Transplantation, St. Orsola-Malpighi Hospital, University of Bologna, Bologna, Italy \\ Correspondence to: Daniele Camboni, MD. Department of Cardiothoracic Surgery, University Medical Center Regensburg, Franz-Josef-Strauss-Allee \\ 11, 93053 Regensburg, Germany. Email: daniele.camboni@ukr.de.
}

\begin{abstract}
Each cannulation strategy for venoarterial extracorporeal membrane oxygenation (VA ECMO) has distinct benefits and drawbacks. In this article, various cannulation strategies including their indications are discussed. The gold standard for cannulation involves peripheral, percutaneous double cannulation utilizing the patient's femoral vein and artery. In emergency situations under mechanical resuscitation, a simple and fast cannulation technique is crucial to reestablish circulation. This is usually performed percutaneously utilizing the femoral approach. However, in cases of anticipated long-term support, such as while awaiting cardiac transplantation, more sophisticated cannulation techniques (e.g., internal jugular vein to left axillary artery, left axillary artery for neuroprotection) are necessary to facilitate mobilization and physical conditioning on VA ECMO. More complicated are cases involving combined respiratory and cardiac failure requiring dual organ support or triple cannulation with an additional venous return cannula resulting in a venoarterio-venous (VAV) configuration. Cases with left ventricular stasis with need for unloading are also highly demanding. Unloading the left ventricle (LV) can be performed in numerous ways, described elsewhere in this issue. However, one particular mode of unloading the $\mathrm{LV}$ is described as a stepwise and cost saving bridge to a durable paracorporeal left ventricular assist device in patients with an uncertain prognosis, which involves implantation of Berlin Heart ${ }^{\circledR}$ EXCOR cannulas with temporary right heart support as an example of quadruple cannulation.
\end{abstract}

Keywords: Extracorporeal life support; cannulation; heart failure

Submitted Jun 25, 2018. Accepted for publication Oct 25, 2018.

doi: 10.21037 /acs.2019.01.03

View this article at: http://dx.doi.org/10.21037/acs.2019.01.03

\section{Introduction}

To answer the title question right away: there is indeed a limit to venoarterial extracorporeal membrane oxygenation (VA ECMO) cannulation. Double cannulation builds the gold standard and quadruple cannulation should be the limit. Venoarterial support is primarily used for cardiogenic shock due to profound left and/or right ventricular failure (1). However, cardiac failure can be complicated by concomitant pulmonary failure or left ventricular stasis. The standard for cannulation involves peripheral cannulation utilizing the femoral vessels. The most viable cannulation technique is the percutaneous Seldinger technique. Based on our clinical experience, we find that whenever the gold standard has to be altered by using additional cannulas or central cannulation, survival outcomes may be decreased. The cannulation strategy naturally depends on the clinical setting. In emergency situations requiring external chest compression, a simple and fast cannulation technique is crucial. However, in cases requiring long-term support, e.g., while awaiting cardiac transplantation, more sophisticated cannulation techniques are necessary to facilitate mobilization on extracorporeal life support (ECLS). Cases with combined respiratory and cardiac failure requiring dual organ support are more demanding as are those with left ventricular stasis and the subsequent need for unloading. Each of these scenarios are discussed in this chapter. 


\section{General aspects of cannulation}

The clinical setting in which a patient undergoes VA ECMO placement is usually challenging. This is particularly the case in VA ECMO assisted mechanical resuscitation (ECPR). It is crucial that the implanting team is welltrained and that systems are in place to minimize severe or fatal complications of cannulation, e.g., vascular laceration or perforation and cardiac tamponade. A cannulation cart with all anticipated equipment including cannulas, wires, drapes and gloves is very helpful in emergency situations. As important as a broad set of cannulas is a variety of guidewires ranging from extra stiff guidewires (e.g., Amplatzer Super Stiff ${ }^{\mathrm{TM}}$, Boston Scientific) to smooth PTFE guidewires (e.g., the angled TERUMO guidewire Radifocus ${ }^{\circledR}$ ). Ultrasound examination of the planned vascular access is helpful in vascular localization. Similarly, echocardiography is useful in facilitating correct placement of the venous cannula into the inferior vena cava or right atrium to optimize drainage. However, especially under ECPR, echocardiographic guidance of guidewire placement is often impossible, and cannula misplacements can occur.

\section{Cannulation technique}

As a general rule, two team members should ideally be present to perform the procedure. Cannulation under local anesthesia is possible for a patient who is spontaneously breathing and alert, but generally patients are sedated and intubated. The technical steps for a standard percutaneous femoral double cannulation are listed in Table 1. It should be noted that the requirement for force during cannulation may be a sign of an evolving complication. Choosing the correct size of cannula diameter can be challenging. For a targeted flow of 3.5-4 L/min in adults, an arterial cannula of $15-17 \mathrm{Fr}$ is sufficient. In the presence of peripheral occlusive arterial disease, it is recommended to use the smaller $15 \mathrm{Fr}$ cannula. It is also recommended to implement distal limb perfusion with a 7.5-9 Fr vascular reinforced sheath (e.g., CruraSave ${ }^{\circledR}$ Femoral-Perfusion Set, freelife medical GmbH, Aachen, Germany) instead of a standard access cannula as it prevents kinking. It is favorable to place a wire distally prior to placement of the actual arterial cannula, since distal flow is usually considerably lower after placement of the arterial cannula. In order to monitor distal leg perfusion Near Infrared Spectroscopy (NIRS) is very useful. It is important to obtain baseline values on both legs prior cannulation to be able to validate leg ischemia. For venous cannulation, generally a $21-23 \mathrm{Fr}$ cannula 35 to $55 \mathrm{~cm}$ long is sufficient in adults.

\section{Double cannulation}

\section{Central cannulation}

For patients with shock post cardiotomy, central cannulation is usually the method of choice. Another important patient group for central cannulation includes newborn and pediatric patients, where peripheral ECLS therapy is not possible due to small vessel calibre. Venous drainage after central cannulation is usually optimal due to larger bore cannulas. A major drawback is that the chest needs to remain open with a high risk of mediastinitis. In addition, cardiopulmonary bypass cannulas are mostly not heparinized which has unclear clinical implications. A hybrid approach with an $8 \mathrm{~mm}$ graft prosthesis anastomosed to the ascending aorta, which is then tunneled externally through the epigastric area and delivered with a 21-23 Fr arterial cannula allows chest closure. Alternative venous drainage can be performed with a long $55 \mathrm{~mm}$ venous cannula inserted via the femoral vein under echocardiographic guidance as described above. Likewise, the right axillary artery can be used for arterial cannulation. In order to avoid neurologic sequelae it may also be advisable to cannulate the left subclavian artery utilizing a tunneled vascular $8 \mathrm{~mm}$ graft (Figure 1). Upper extremity hyperperfusion can occur following cannulation of the axillary artery (2). From our experience, this rarely poses a clinical problem. In such cases, banding of the axillary artery can help to decrease distal perfusion. Venous drainage can also be performed through the right internal jugular vein via a cannula with multiple side holes placed with its tip in the inferior vena cava. This enables patient mobilization on VA ECMO. Finally, the ability to unload the left ventricle (LV) in post cardiotomy patients is facilitated since the chest is already open with a variety of venting possibilities (Figure 2).

\section{Peripheral cannulation}

Peripheral cannulation is likely the most commonly applied approach to establish mechanical circulatory support with VA ECMO. The right femoral vein is recommended for venous cannulation with its tip lying in the right atrium. The choice of diameter of the venous cannula depends on patient size as well as the clinical circumstances (e.g., smaller cannulas are preferable in emergency situations). A 21-23 Fr venous cannula in adults is the most common 
Table 1 Technical aspects

1. 'Team time out' is recommended to ensure that all equipment and personnel are in place in order to minimize potential complications. Two units of blood should be available but not at the bedside in cases of elective cannulation

2. The patient must be positioned appropriately (e.g., supine or Trendelenburg position in cases of neck cannulation)

3. Standard preparation under sterile conditions must be carried out. A portable ultrasound is helpful in localizing the relevant vascular anatomy

4. A Seldinger technique is used with a 20-gauge needle. A short guidewire can be used initially to access the vessel. Placement of a vascular sheath can be helpful where there is uncertainty. The order of arterial vs. venous cannulation is usually not important. The first dilator is introduced at the beginning enabling a subsequent guidewire change (e.g., $1.5 \mathrm{~m}$ long 0.035 guidewire for venous cannulation as usually comes with the cannula set). A Terumo guidewire can be useful where there is kinking of the vessels. The Terumo guidewire should be replaced using a long sheath (e.g., pigtail) to introduce a stiffer guidewire if needed prior to placement of the cannula

5. In the event of venous cannulation and availability of echocardiography, positioning of the guidewire in the right atrium should be confirmed by pulling and pushing the wire to increase visibility. X-ray imaging can also be used to visualize the cannulation procedure. In case Terumo and stiffer guidewires are needed fluoroscopy is recommended to avoid vascular injuries

6. A small incision is usually not necessary, however may be useful in some patients, particularly males

7. Dilators should be advanced while pulling the guidewire slightly to keep the wire on tension. It is important to be gentle and to not use force while advancing the dilators. After every dilator change, the guidewire should be replaced

8. When the dilator is exchanged, it is crucial to manually compress the puncture site to avoid excessive bleeding

9. A heparin bolus of $50-100 \mathrm{U} / \mathrm{kg}$ should be given after secure vessel access

10. All necessary dilators from the cannula kit should be used up to the appropriate size. One should not over-dilate do avoid bleeding at the puncture site

11. One needs to ensure that the introducer is beyond the cannula tip by at least a few centimeters. When advancing the cannula, it is extremely important to advance the introducer with the cannula as a single unit. As with the dilators, it is important to apply some gentle pulling pressure on the guidewire for proper placement of the cannula. Slight rotational movements can help with cannula placement

12. When the venous cannula is in the right atrium as demonstrated on echocardiography, the introducer and guidewire are simultaneously removed, backflow of blood is confirmed and the venous cannula is clamped. It is important to minimize blood loss best as possible during this step

13. The arterial cannulation follows the same principles

14. It is important to ensure that the tubing is free of air bubbles, particularly at the junctions of the connectors, and to connect the cannulas to the ECMO tubing. Flushing cannulas before connection to the ECMO circuit to wash out possible thrombus formations is strongly recommended

15. One should commence therapy gently by augmenting flow to the desired rate. $3-4.5 \mathrm{~L} / \mathrm{min}$ is our preferred flow rate. Other centers promote a higher flow rate strategies with flows higher than $4.5 \mathrm{~L} / \mathrm{min}$ which has as associated risk of left ventricular distension secondary to afterload increase

16. Post cannulation, imaging is crucial to ensure that any misplacements are corrected promptly

size from our experience, however the routine use of bigger and smaller cannulas is described as well. The rationale for use of the right side relates to the relatively vertical course of the right femoral and common iliac veins into the rightsided inferior vena cava. The left common iliac vein, on the other hand, crosses the vertebral column and aorta to reach the inferior vena cava, resulting in greater risk from left-sided venous cannulation. For arterial cannulation, the contralateral side is recommended, however, unilateral cannulation may be unavoidable. Generally, 15-17 Fr arterial cannulas are sufficient in adults. In most cases a percutaneous technique is possible utilizing a Seldinger techniques and only in rarer cases is an open cannulation technique required. 
A

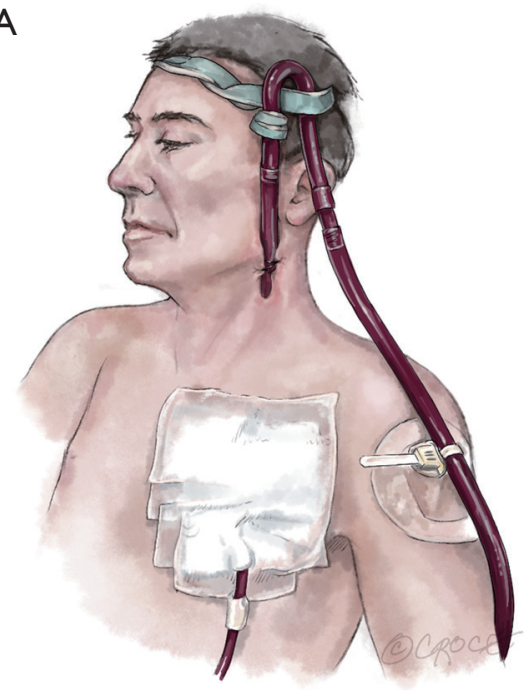

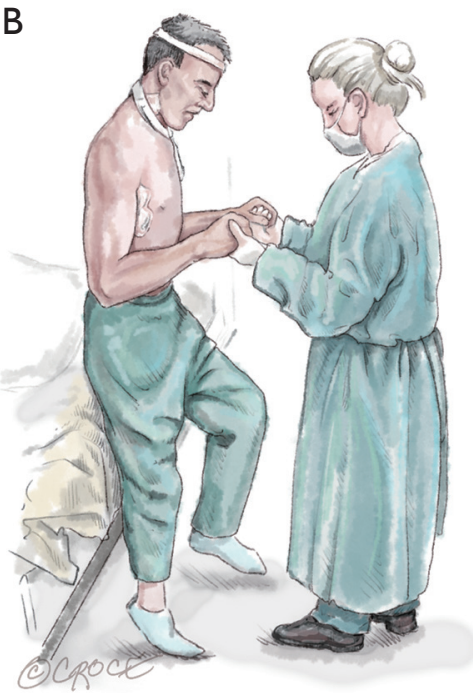

Figure 1 Cannulation strategy for patients supported waiting for a suitable donor heart. (A) Tunneled 23 Fr arterial cannula inserted into the left axillary artery through a sutured $8 \mathrm{~mm}$ vascular graft and $35 \mathrm{~cm} 23 \mathrm{Fr}$ venous cannula inserted percutaneously into the left jugular vein under echocardiographic guidance; (B) patient on VA ECMO under physical exertion on support waiting for several months for a suitable donor heart. VA ECMO, venoarterial extracorporeal membrane oxygenation.

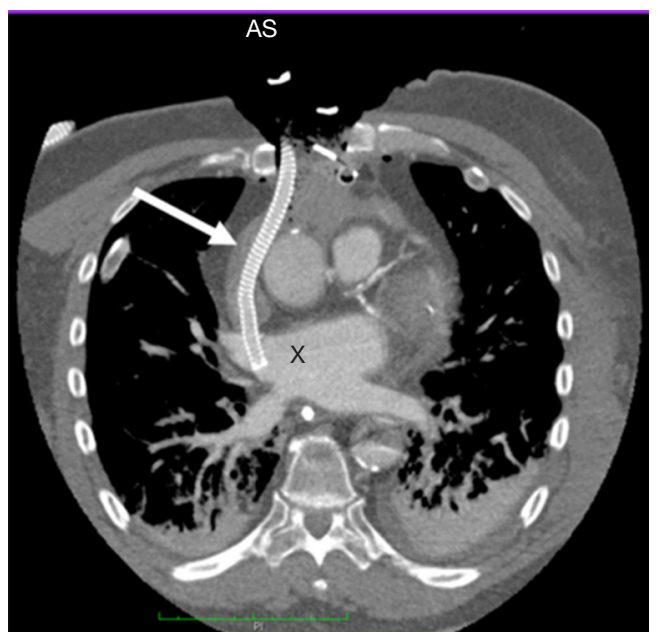

Figure 2 Venting the left atrium in a postcardiotomy patient with an open chest. X: left atrium; white arrow: cannula. Note the open chest which is closed in a sterile fashion with a drape. Venting is usually only necessary for a few days; on closing the chest venting can either be stopped or therapy is modified to another support system.

\section{Triple cannulation}

A major drawback of peripheral cannulation is the risk of a so-called Harlequin or North-South syndrome which may evolve as a consequence of upper body hypoxemia due to concomitant lung failure or incorrect ventilator settings. A watershed forms inside the aorta when welloxygenated retrograde blood from the femoral arterial cannula meets poorly oxygenated blood from the LV. The under-saturated blood may result in malperfusion of the coronary and cerebral vasculature (3). Early detection and treatment of this requires meticulous monitoring of right upper extremity arterial blood gases. These patients suffering from cardiac and pulmonary failure with an upper body hypoxemia on VA ECMO may benefit from an additional venous infusion cannula if ventilation settings and pulmonary function cannot be improved by other measures. The additional $15-21 \mathrm{Fr} 35 \mathrm{~cm}$ long venous return cannula is usually inserted into the right internal jugular vein with the tip in the superior vena cava at a minimum distance to the inferior vena cava cannula of $15 \mathrm{~cm}$ to minimize recirculation. This type of support is called veno-arterio-venous (VAV) support. Here, part of the arterial outflow is directed toward the right atrium and to the pulmonary vasculature thus oxygenating the coronary arteries and the upper body more efficiently (Figure 3) (4). The amount of arterial outflow to the venous side can be altered with a Hoffman Clamp if systemic circulatory support is critically lowered. By applying a clamp to the backflow cannula hemolysis may occur resulting in a blood product requirement. Use of venous tubing with higher 

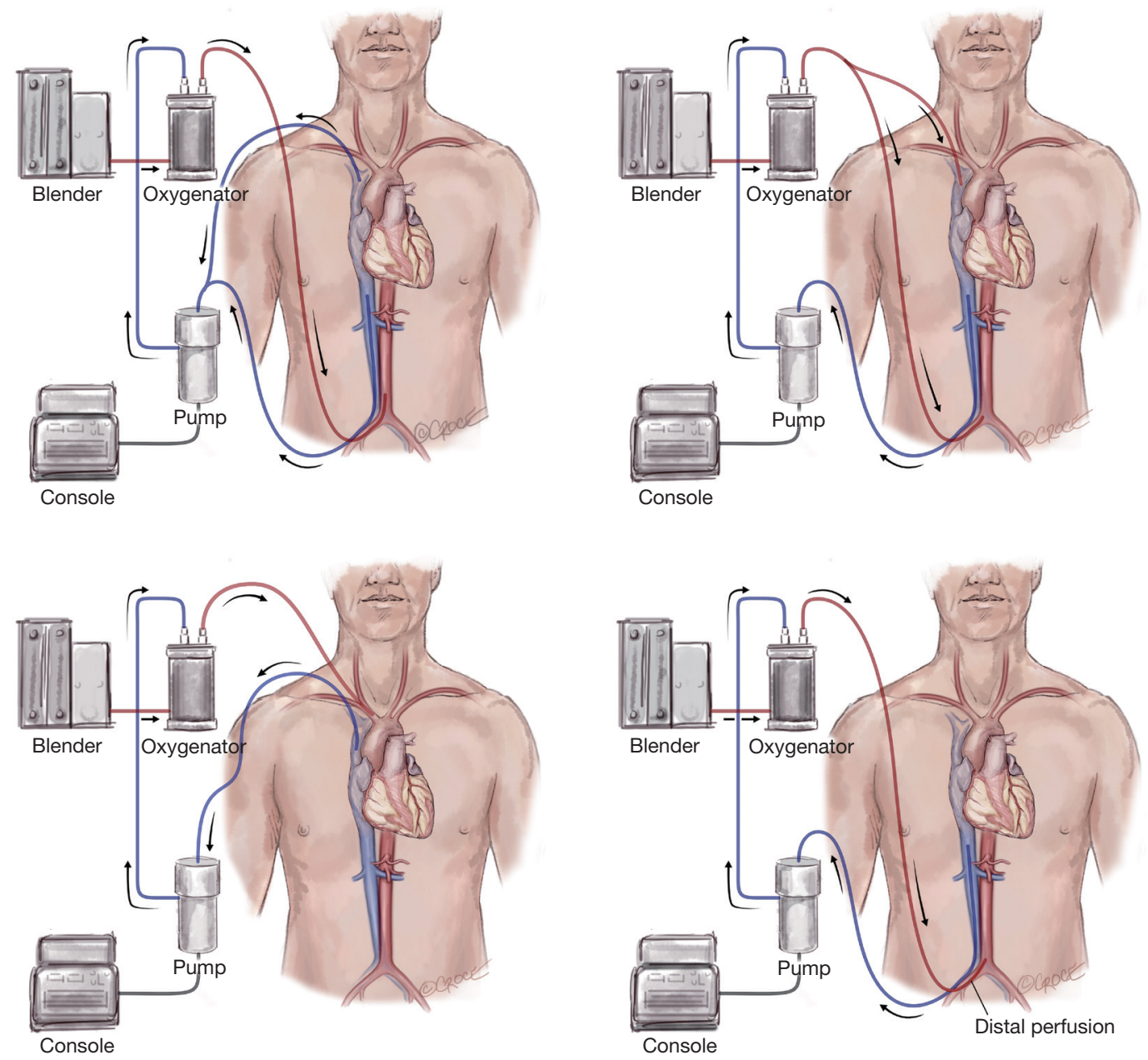

Figure 3 Different modes of VA ECMO: VA, VVA, VAA, VAV. VA ECMO, venoarterial extracorporeal membrane oxygenation; VVA, veno-veno-arterial; VAA, veno-arterio-arterial; VAV, veno-arterio-venous.

resistance may lower hemolysis and secondary transfusion requirements.

Triple cannulation may also be necessary in cases of insufficient venous drainage with one peripheral cannula in place. By adding an additional drainage cannula, venous drainage can be improved. However, the reason for insufficient venous drainage is usually volume depletion or cannula misplacements, which can be easily corrected by volume administration or replacement of the cannula. Some physicians refer to this type of cannulation as veno-veno-arterial (VVA) support (5). On the other side veno-arterio-arterial (VAA) support is an additional arterial backflow cannula in cases of high resistance backflow. However, the limiting factor for blood flow doesn't usually relate to the arterial cannula; it is typically a venous drainage problem. Overall, the need for addition of a venous (VVA) or arterial cannula (VAA) for improved drainage or backflow is rare in our clinical experience. The application of an additional venous backflow cannula in cases of concomitant severe lung failure on VA ECMO (i.e., for VAV support) is more common.

\section{Triple cannulation to unload the LV on VA ECMO}

VA ECMO does not inherently decompress the LV, since even with full veno-arterial support of 4-5 L/min there will be blood return into the LV. This can be problematic in the setting of poor ventricular function and especially in high-grade aortic valve incompetence. VA ECMO increases afterload significantly impairing cardiac output of the failing 
$\mathrm{LV}$. The LV can consequently distend and pulmonary edema may develop. In addition, elevated left ventricular filling pressures may decrease myocardial perfusion resulting in further damage to the already injured LV. Therefore, every patient must be conscientiously monitored for signs of left ventricular distension and pulmonary edema. Although unloading the ventricle does not necessarily improve outcomes (6), many centers promote early decompression of the LV (7). Some general aspects of triple cannulation to decompress the $L V$ are described here however further discussion regarding left ventricular decompression in the setting of VA ECMO for refractory heart failure is detailed elsewhere in this issue.

Unloading the LV may be carried out by placement of a third cannula or by addition of a second mechanical circulatory support system. The third cannula can be placed percutaneously into the main pulmonary artery to reduce blood flow reaching the LV. Since only the left ventricular filling is reduced, the degree of decompression is rather low and blood stasis can still occur. The same is true for a percutaneous trans-septal cannula (e.g., TandemHeart cannula) advanced into the left atrium. In this setting, blood stasis in the LV is again not sufficiently treated and only volume overload is reduced.

Another option is to place a cannula surgically into the $\mathrm{LV}$ through the right upper pulmonary vein or directly into the $L V$ via the left ventricular apex similar to a transapical TAVR technique. The latter may complicate later placement of a left ventricular assist device. It should be emphasized that this mode of placement of an additional cannula is a surgical intervention requiring sternotomy or thoracotomy in a highly vulnerable patient with a significant risk of bleeding. Therefore, benefits and risks including those relating to the likelihood of myocardial recovery should be weighed against each other before venting the $\mathrm{LV}$ surgically. Moreover, there is no clear evidence beyond physiologic decompression of the LV that unloading the LV leads to improved survival. Optimization of perfusion by lowering ECLS flow to $3-4 \mathrm{~L} / \mathrm{min}$ and permitting a mean arterial pressure of $45-50 \mathrm{mg}$ in situations of no cardiac output, with close monitoring of acid-base balance and lactatemia, provides a reasonable alternative and renders venting procedures nearly unnecessary. Only where persistent pulmonary edema with left ventricular stasis and sludge formation despite optimized ventilation, fluid management and afterload reduction are venting the $L V$ is absolutely necessary (8).

Other methods of unloading the LV include use of an intra-aortic balloon pump and the more recently utilized Impella $^{\circledR}$ (Abiomed, USA).

\section{Quadruple cannulation}

With the increasing use of VA ECMO under resuscitation (ECPR) on an international level, a new patient group is being generated. This includes resuscitated patients in biventricular failure too good for withdrawal of therapy and too unwell for heart transplantation or implantation of a durable left ventricular assist device. This sick patient population with biventricular failure and concomitant multi-organ dysfunction can be stabilized by quadruple cannulation as a bridge to cardiac transplantation or left ventricular assist device implantation.

Since total artificial heart implantation is still associated with unsatisfactory outcomes, transitioning to a more cost-effective durable left ventricular assist device can be achieved by a stepwise approach. Following primary peripheral VA ECMO stabilization and end-organ resuscitation with persisting biventricular failure and left ventricular stasis, a four-step procedure can be applied resulting in paracorporeal left ventricular assist support (Figures 4-6). After excluding severe contraindications (e.g., malignancies, brain hemorrhage), surgical implantation of the paracorporeal $\mathrm{EXCOR}^{\circledR}$ cannulas can be performed. This is done via a median sternotomy suturing the inflow cannula to the left ventricular apex and the outflow cannula to the ascending aorta, after which both cannulas are passed externally. This surgical procedure can be carried out under ECLS flow or rendered more secure with the aid of standard cardiopulmonary bypass. Concomitant right ventricular failure is treated by suturing an $8 \mathrm{~mm}$ vascular graft to the pulmonary artery, which is then also externalized and cannulated with a $21 \mathrm{Fr}$ arterial cannula. One circuit is built with the left-sided EXCOR cannulas and the other with the femoral venous cannula and the newly inserted pulmonary artery cannula. This temporary right heart system can be subsequently removed without re-opening the chest. Both EXCOR cannulas are attached to a centrifugal pump (Rotaflow, Maquet Rastatt, Germany) to minimize costs in certain situations of uncertain prognosis. If an oxygenator is needed in the right-sided support system due to an impaired pulmonary function it can be easily introduced into the circuit. The oxygenator can then be removed when lung function improves. When weaning from the temporary right ventricular support system is successfully achieved, it can be easily removed at the bedside in the intensive care unit. The 

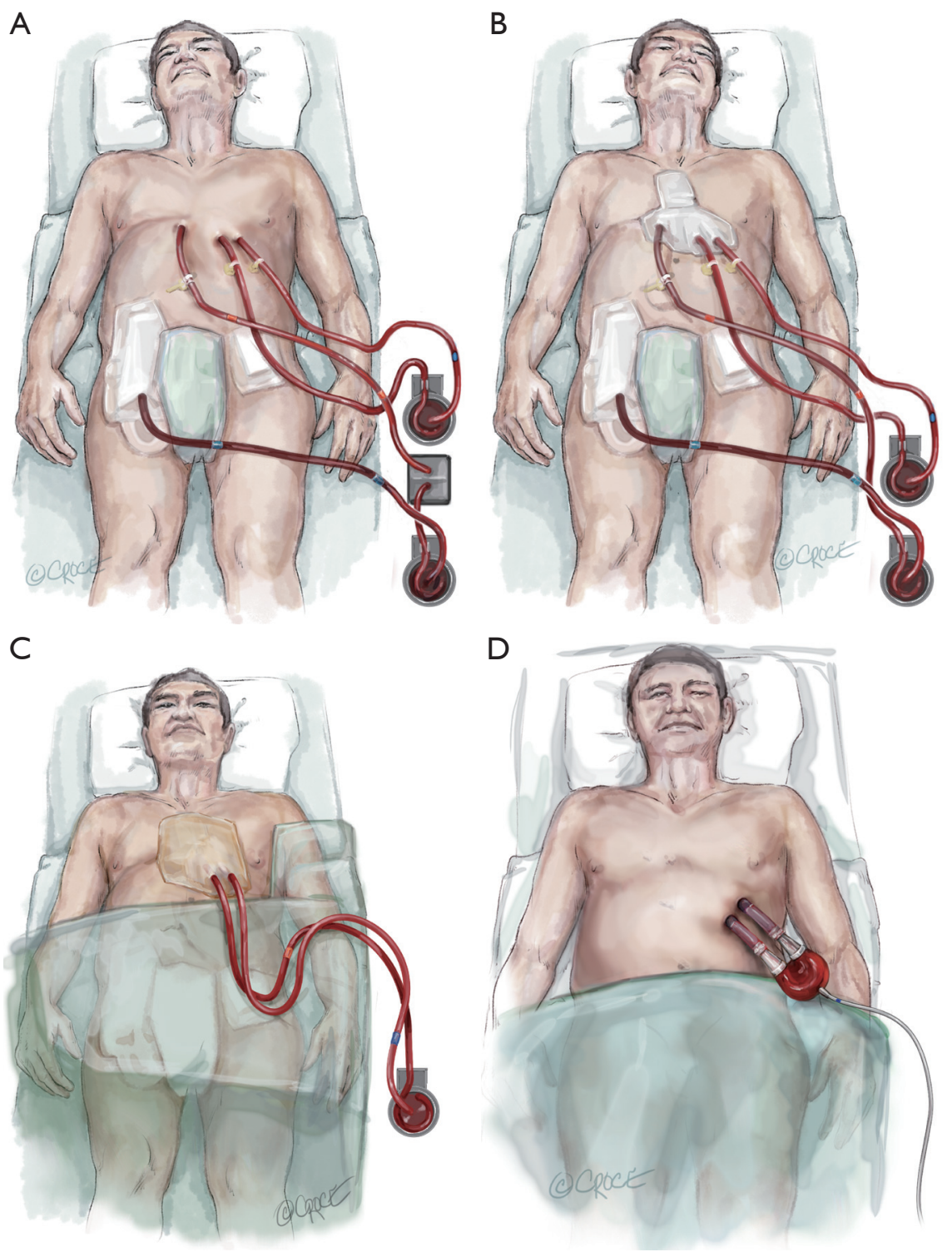

Figure 4 Bridge to durable LVAD. (A) Step 1: after exclusion of contraindications (e.g., brain damage after ECPR) Berlin Heart EXCOR cannulas are implanted. The EXCOR cannula in the left ventricle and the ascending aorta facilitates unloading of the left ventricle avoiding pulmonary congestion and reaching higher flows than typical implantable left ventricular assist devices. The right heart can be supported with an $8 \mathrm{~mm}$ graft sutured to the pulmonary artery, which is externalized. Venous drainage occurs from the inferior vena cava. Thereby, the chest can be closed without the need to reopen the chest. An oxygenator can be inserted in case of poor pulmonary function; (B) step 2: weaning the temporary right heart support. The oxygenator should be out of the circuit; (C) step 3: removal of the right heart support after successful weaning of the temporary support system; (D) step 4: attachment of the Berlin Heart EXCOR chambers to the previously implanted cannulas.

final step involves connection of the EXCOR chambers to the previously implanted EXCOR cannulas. This can also be accomplished at the bedside in the intensive care unit under sterile conditions. The connection of EXCOR chambers takes 1 to 2 minutes with resulting circulatory depression.
The patient may subsequently mobilize, be discharged and bridged to cardiac transplantation. Our institution treated 7 patients over the last decade in such a manner with a $71 \%$ survival to discharge (9). The main advantage of this four-step approach in this poor prognosis patient population 
is cost saving in comparison to implant directly a durable, implantable left ventricular assist device with some sort of temporary right heart support. Another advantage of this four-step approach is that higher flows can be achieved in comparison to durable left ventricular assist devices

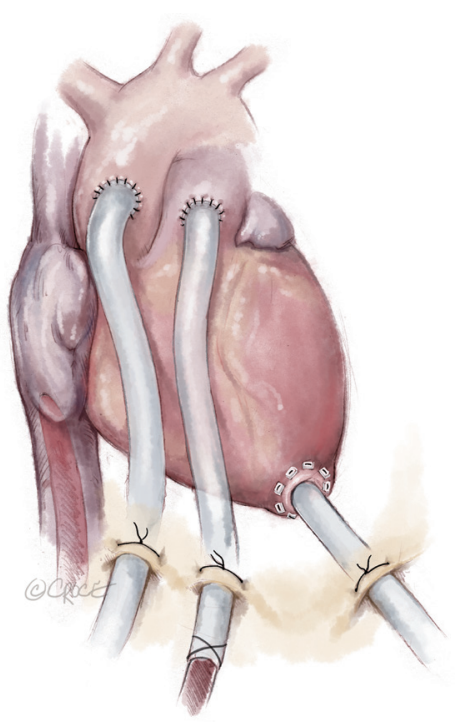

Figure 5 Open chest illustrated with a graft sutured to the pulmonary artery, implanted EXCOR cannulas through the apex of the left ventricle, vascular graft to the ascending aorta with an inserted cannula and draining cannula in the inferior vena cava. in a situation with a higher flow demand in this patient population.

\section{Discussion}

The vast majority of patients in acute or acute on chronic heart failure can be cannulated percutaneously in a femoralfemoral fashion. Post-cardiotomy patients preferably remain on central cannulation with the major drawback of an increased risk for mediastinitis and a possibly higher risk for neurologic injury (own data, publication in process). In addition, a third cannula increases the complexity of the VA ECMO and therefore all conservative measures should be applied to avoid complicating the circuit. This applies for the VAV approach for pulmonary and circulatory support as well as the VVA or VAA approaches. The addition of a third cannula to unload the LV is rarely needed in our experience and afterload reduction in addition to lower flows of 3-4 L/min are generally sufficient to avoid left ventricular distension. Nevertheless, there are patient cohorts such as those with aortic insufficiency who certainly need left ventricular decompression. Quadruple cannulation or differentiated biventricular support remains an option to bridge certain patients to durable left ventricular support. Thus, in the setting of VA ECMO therapy for heart failure, less is more, however it is crucial to know varied cannulation strategies for some selected patients.
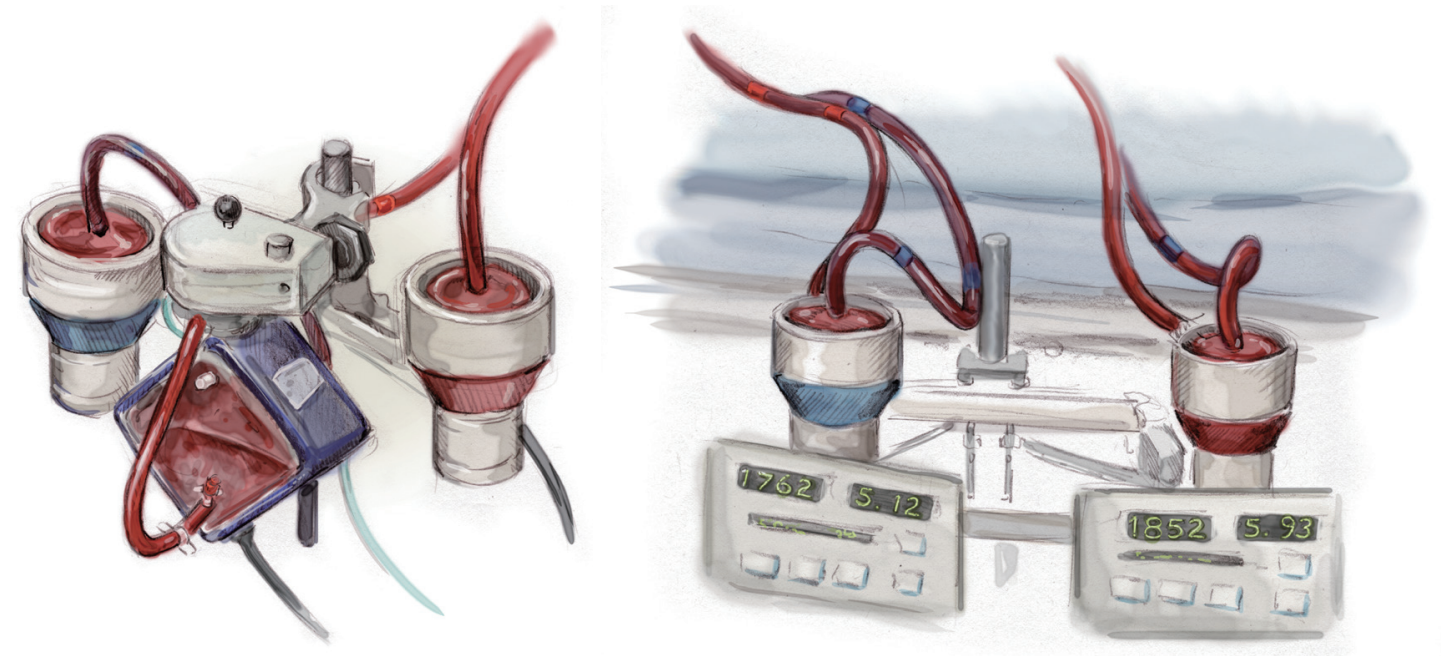

Figure 6 Biventricular support with and without an oxygenator. Note the significantly lower flow on the left side (bright red blood = arterial blood) of the picture $(5.12 \mathrm{~L} / \mathrm{min}$ compared to $5.93 \mathrm{~L} / \mathrm{min})$ representing appropriate right ventricular support to avoid pulmonary congestion in the setting of biventricular support. Note, a flow of $5.9 \mathrm{~L} / \mathrm{min}$ is barely possible in durable, implantable left ventricular assist devices. 


\section{Acknowledgements}

None.

\section{Footnote}

Conflicts of Interest: The authors have no conflicts of interest to declare.

\section{References}

1. Guenther SP, Brunner S, Born F, et al. When all else fails: extracorporeal life support in therapy-refractory cardiogenic shock. Eur J Cardiothorac Surg 2016;49:802-9.

2. Chamogeorgakis T, Lima B, Shafii AE, et al. Outcomes of axillary artery side graft cannulation for extracorporeal membrane oxygenation. J Thorac Cardiovasc Surg 2013;145:1088-92.

3. Hoeper MM, Tudorache I, Kühn C, et al. Extracorporeal membrane oxygenation watershed. Circulation 2014;130:864-5

4. Choi JH, Kim SW, Kim YU, et al. Application of veno-

Cite this article as: Camboni D, Philip A, Schmid C, Loforte A. Double, triple and quadruple cannulation for veno-arterial extracorporeal membrane oxygenation support: is there a limit? Ann Cardiothorac Surg 2019;8(1):151-159. doi: 10.21037/ acs.2019.01.03 arterial-venous extracorporeal membrane oxygenation in differential hypoxia. Multidiscip Respir Med 2014;9:55.

5. Napp LC, Kühn C, Hoeper MM, et al. Cannulation strategies for percutaneous extracorporeal membrane oxygenation in adults. Clin Res Cardiol 2016;105:283-96.

6. Truby LK, Takeda K, Mauro C, et al. Incidence and Implications of Left Ventricular Distention During Venoarterial Extracorporeal Membrane Oxygenation Support. ASAIO J 2017;63:257-65.

7. Pappalardo F, Schulte C, Pieri M, et al. Concomitant implantation of Impella ${ }^{\circledR}$ on top of veno-arterial extracorporeal membrane oxygenation may improve survival of patients with cardiogenic shock. Eur J Heart Fail 2017;19:404-12.

8. Meani P, Gelsomino S, Natour E, et al. Modalities and Effects of Left Ventricle Unloading on Extracorporeal Life support: a Review of the Current Literature. Eur J Heart Fail 2017;19 Suppl 2:84-91.

9. Rupprecht L, Camboni D, Philipp A, et al. Further options and survival results after failure following ECLS implantation. J Cardiovasc Surg (Torino) 2019;60:128-35. 